The future of complementary and integrative medicine research international perspectives

Witt, Claudia M ; Barth, Jürgen ; Canella, Claudia

DOI: https://doi.org/10.1159/000381512

Posted at the Zurich Open Repository and Archive, University of Zurich ZORA URL: https://doi.org/10.5167/uzh-116797

Journal Article

Published Version

Originally published at:

Witt, Claudia M; Barth, Jürgen; Canella, Claudia (2015). The future of complementary and integrative medicine research - international perspectives. Forschende Komplementärmedizin, 22(2):125-127.

DOI: https://doi.org/10.1159/000381512 


\title{
The Future of Complementary and Integrative Medicine Research - International Perspectives
}

\author{
Claudia M. Witt Jürgen Barth Claudia Canella \\ Institute for Complementary and Integrative Medicine, University Hospital Zurich and University Zurich, Zurich, Switzerland
}

\section{Background of the Symposium}

In January 2014, Claudia Witt became the new director of the Institute for Complementary and Integrative Medicine at the University Hospital Zurich, based at the University of Zurich. December 1, 2014 she gave her public inauguration lecture as Chair of the institute (fig. 1). On the same day, the institute organized an international symposium on 'The Future of Complementary and Integrative Medicine Research', which took place at the University Hospital Zurich. The aim of this half-day symposium was to provide interdisciplinary and international perspectives, which have the potential to bring forward future complementary and integrative medicine research. After the opening speeches by the hospital $\mathrm{CEO}$, the vice president of the university, and the dean of the medical school, 7 speakers from 3 countries (UK, USA, and Switzerland; fig. 2), including Claudia Witt, provided insights into new research developments in clinical, placebo, and comparative effectiveness research as well as systems biology. Their presentations can be viewed at www.iki.usz.ch/forschung/seiten/praesentationen.aspx.

\section{Symposium Summary}

\section{Brian Berman: '20 Years of the Cochrane Complementary}

Medicine Field - Where Do We Stand?'

Speaking about '20 Years of the Cochrane Complementary Medicine Field - Where do we stand?', Brian Berman, Professor of Family and Community Medicine and Coordinator of the Cochrane CAM field at the University of Maryland, School of Medicine in Baltimore, provided an overview of the history and current state of the Cochrane Complementary Medicine Field [1]. The aim of the Cochrane Collaboration is preparing, maintaining, and disseminating systematic reviews on the effects of health care interventions. The collaboration consists of 31,000 contributors from more than 120 countries, 39 Cochrane centers, 53 collaborative review groups, 10 subfields, 16 method groups, and 1 consumer network. Cur- rently, there are about 57,500 CAM trials from all over the world registered in the Cochrane Central Register of Controlled Trials and about 690 systematic CAM reviews in the Cochrane Database. Berman presented a few examples of systematic CAM reviews before concluding with future challenges, such as conducting reliable subgroup analyses (e.g., individual patient data meta-analysis or network meta-analysis) and systematic reviews on complex interventions as well as defining what can be considered as scientifically rigorous or relevant to real-world practice of medicine.

\section{Irving Kirsch: 'Placebo Effects in Complementary and \\ Conventional Medicine'}

Irving Kirsch, Professor emeritus of medicine at the University of Connecticut, University of Hull, Plymouth University, and associate director of Placebos Studies and Therapeutic Encounter (PiPS) at the Beth Israel Deaconess Medical Center in Harvard Medical School provided insights into the most recent research on placebo effects in complementary and conventional medicine. He introduced his presentation with the distinction between placebo response, which is the general reaction to a placebo, and the placebo

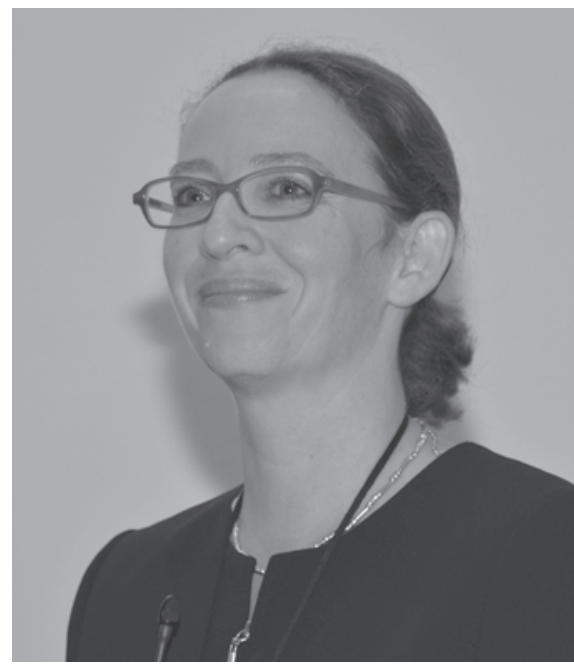

Fig. 1. Claudia M. Witt, Chair for Complementary and Integrative Medicine at the University of Zurich.
Claudia M. Witt, MD, MBA 

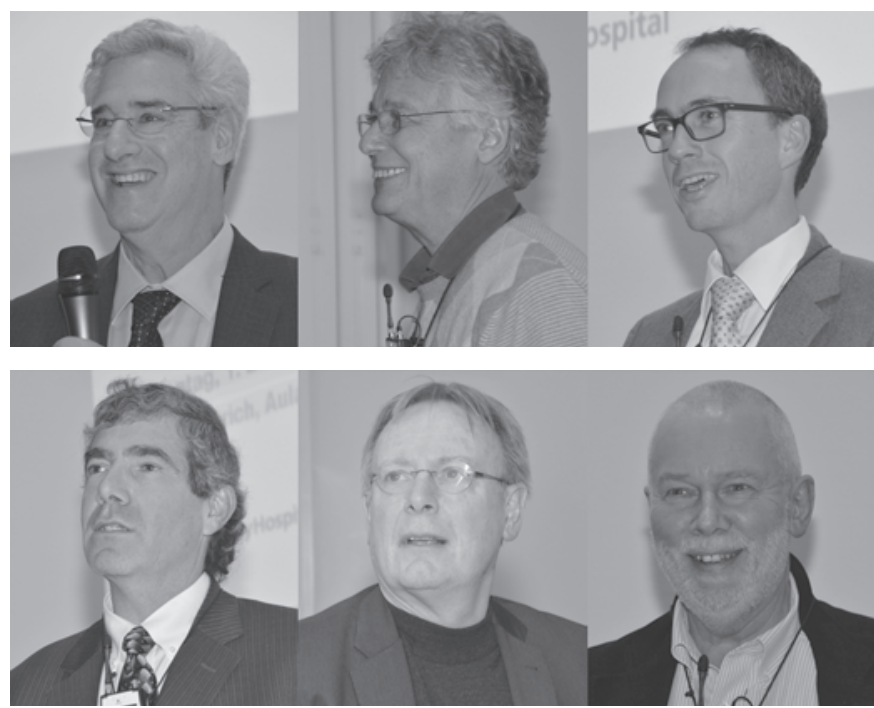

Fig. 2. Speakers of the international symposium 'The Future of Complementary and Integrative Medicine Research'. From left to right: (top) Brian Berman, Irving Kirsch, Jürgen Barth; (bottom) Steven Woolf, Jan van der Greef, Paul Dieppe.

effect, which corresponds with the effect of a placebo compared to usual treatment and to the natural history of a disease. Supported by several examples of meta-analyses [e.g., 2, 3] and his own research [e.g. 4], Kirsch showed that both in complementary and conventional medicine the placebo effect as well as the medical treatment effect depend on the condition and on various characteristics of the treatment, such as the color of the placebo pill, dose, strength of 'drug', brand name, price, and therapeutic relationship. To give one example: 4 placebo pills a day resulted in a higher response rate than 1 placebo pill a day [5]. According to Kirsch, it is therefore necessary to examine within-group pre-post changes in both the active treatment and placebo groups as well as report betweengroup differences in clinical trials and meta-analyses.

\section{Jürgen Barth: 'Patient-Doctor-Scientist Interaction: Impact on}

\section{Treatment Outcome'}

In his presentation on 'Patient - Doctor - Scientist Interaction: Impact on Treatment Outcome’, Jürgen Barth, Research Director at the Institute for Complementary and Integrative Medicine, outlined 3 perspectives that might influence the outcomes of integrative medicine in research and practice. Patient expectations toward treatment are an important patient characteristic with an impact on treatment outcomes [6], but they do not necessarily match with the expectations of health professionals [7]. Thus, communication between physicians and patients in practice might also contribute to better health outcomes, especially if patients perceive the communication as helpful [7]. Patient expectations fuelled by health professionals might therefore also influence outcomes in placebo research. The scientist might also contribute to treatment effects by own expectations (i.e. allegiance). The allegiance towards a specific treatment might bias the research results and overestimate treatment effects [8]. Barth concluded that future research should more often use patient expectations as predictor of treatment response, and that better scales are needed for valid assessment. Since good communication might result in improved working alliance, a critical evaluation of the working alliance during the entire treatment seems necessary. Allegiance effects and biases can be lowered by collaborative trials and by disclosure statements of scientists and doctors.

\section{Paul Dieppe: 'The Evaluation of Health and Wellbeing 'on "wellbeing",}

Paul Dieppe, Professor for Health and Wellbeing at the Medical School University of Exeter, shed light on 'The Evaluation of Health and Wellbeing 'on "wellbeing", discussing the definition of the term, the different approaches to access the topic, and assessment methods used in different disciplines. In this context, Dieppe introduced some ideas by the ancient Greeks, contrasting 'eudaimonia' (fulfilling your potential to the benefit of everyone in society) and 'hedonism' (personal happiness); he pointed out that in today's culture most discussions on wellbeing concentrated on personal happiness rather than on the benefits of groups or communities. Many different aspects of our cognitions, emotions, and behaviors as well as the environment and circumstances we live in are used as basis to assess wellbeing, and several different disciplines claim to have suitable assessment methods. According to Dieppe, there are 5 different domains entailing wellbeing: mental health, physical health, social circumstances, spiritual wellbeing, and possessions, for which both subjective (self-report) and more objective measures are used. Dieppe's colleagues Myles-Jay Linton and Antonieta Medina-Lara are reviewing and classifying all the different ways of measuring wellbeing for the benefit of researchers. However, Dieppe recommended that we need to move from a pathological to a salutogenic understanding of health and wellbeing (the generation of health as promoted by Aaron Antonovsky), thereby focusing on manageability, meaningfulness, and comprehensibility $[9,10]$. He concluded that the selected method to evaluate wellbeing should correspond with the research approach, and characterized wellbeing as holistic concept entailing body, mind, and spirit.

\section{Jan van der Greef: 'Bridging Western and Chinese Medicine with Systems Biology'}

Jan van der Greef, Professor of Analytical Bioscience at Leiden University and Principal Scientist at TNO, promoted 'Bridging Western and Chinese Medicine with Systems Biology', introducing a 'systems view on life'. He stated that more than $90 \%$ of drugs only work in $30-40 \%$ of people in today's health care. As a result, van der Greef suggestd a shift from a 'one-size fits all' to 'personalized' medicine, or, in terms of systems biology, to Western biomarkerguided medicine supported by the phenomenological approach of Chinese Medicine in diagnosis [11-13]. Further, in his opinion it is mandatory to apply comparative effectiveness research in realworld settings. Such an approach implies analyzing complex interdependences of the physical, mental, emotional, social, and spiritual aspects in relation to environmental circumstances of every individual concerned. Van der Greef concluded that personalized medicine must change its focus from symptoms, especially in chronic disease to resilience, against the background of social, physical, and emotional challenges. 


\section{Claudia Witt: 'Comparative Effectiveness Research (CER) in Complementary and Integrative Medicine'}

With her presentation of 'Comparative Effectiveness Research (CER) in Complementary and Integrative Medicine', Claudia Witt, Professor of Medicine and Director of the Institute for Complementary and Integrative Medicine, provided insights into recent developments in a relatively new research field. She focused on randomized trials and outlined that the eligibility criteria of study participants, the flexibility of the interventions, and the type of outcome measures are key criteria to distinguish between randomized controlled trials providing information on the efficacy of a treatment (evidence from ideal study settings) and randomized pragmatic trials providing evidence on the effectiveness of an intervention (evidence from a usual care study setting). Witt showed that acupuncture research has already made unique contributions to comparative effectiveness research (CER) [14] by developing guiding recommendations $[15,16]$. The most recent recommendations even try to close the gap between CER and 'omics'.

Steven Woolf: 'The Impact of Stakeholder Engagement - Lessons Learned from Health Services and Public Health Research'

Steven Woolf, Professor of Family Medicine and Director of the VCU Center on Society and Health at Virginia Commonwealth University in Richmond, gave a lecture on 'The Impact of Stakeholder Engagement - Lessons Learned from Health Services and Public Health Research'. He presented 2 research projects involving stakeholder engagement that had been conducted by his institution [17].

He first noted the emergence of an international trend in health research that emphasizes deeper engagement of the 'affected party.'
In clinical research, this includes an emphasis on shared decisionmaking and patient-centered outcome research. A hallmark of this approach is a commitment to stakeholder engagement at every stage of a research project. Woolf described in detail the process of patient engagement that was undertaken in a pilot study on how patients approach decisions about cancer screening. A second example involved community engagement, in which residents of a community in the East End of Richmond helped set population health priorities and developed new causal models about the role of social determinants of health.

Woolf concluded that stakeholder engagement means first of all forging a coequal partnership and a transition from research subjects to co-investigators.

\section{Conclusion}

There is already a decent amount of clinical research on complementary and integrative medicine. However, the discussion made clear that the bio-psychosocial understanding of and approach to disease in complementary and integrative medicine goes along with a large amount of complexity that has to be considered when designing future trials. Recent research on placebo as well as new approaches, such as systems biology and CER, could be helpful to guide future research.

\section{Acknowledgment}

We thank Maren Schoenfeld for organizing the Symposium and Jürg Lendenmann for the photos.

\section{References}

1 Cochrane CAM Field. www.compmed.umm.edu/ cochrane_about.asp (accessed 11.03.2015).

2 Howick J, Friedemann C, Tsakok M, Watson R, Tsakok T, Thomas J, Perera R, Fleming S, Heneghan C: Are treatments more effective than placebos? A systematic review and meta-analysis. PLoS One 2013;8:e62599.

3 Hróbjartsson A, Gøtzsche PC: Is the placebo powerless? An analysis of clinical trials comparing placebo with no treatment. N Engl J Med 2001;344:1594-1602.

4 Kirsch I: The emperor's new drugs: medication and placebo in the treatment of depression; in Rosenthal W (ed): Handbook of Experimental Pharmacology. Springer, New York, 2014, pp 291-303.

5 Ford AC, Moayyedi P: Meta-analysis: factors affecting placebo response rate in the irritable bowel syndrome. Aliment Pharmacol Ther 2010;32:144-158.

6 Linde K, Witt CM, Streng A, Weidenhammer W, Wagenpfeil S, Brinkhaus B, Willich SN, Melchart D: The impact of patient expectations on outcomes in four randomized controlled trials of acupuncture in patients with chronic pain. Pain 2007;128:264-271.

7 Rakel D, Barrett B, Zhang Z, Hoeft T, Chewning B, Marchand L, Scheder J: Perception of empathy in the therapeutic encounter: effects on the common cold. Patient Educ Couns 2011;85:390-397.
8 Munder T, Brütsch O, Leonhart R, Gerger H, Barth J: Researcher allegiance in psychotherapy outcome research: an overview of reviews. Clin Psychol Rev 2013; 33:501-511.

9 Lundberg O, Nyström Peck M: A simplified way of measuring sense of coherence. Experiences from a population survey in Sweden. Eur J Public Health 1995;5:56-59.

10 Benz C, Bull T, Mittelmark M, Vaandrager L: Culture in salutogenesis: the scholarship of Aaron Antonovsky. Blob Health Promot 2014;4:16-23.

11 Sanders S, Fan TP, Briggs J, Liu L, Lu A, van der Greef $\mathrm{J}, \mathrm{Xu} \mathrm{A}$ : The art and science of traditional medicine part 1: TCM today - a case for integration. Science 2014;346:1569.

12 Van der Greef: All systems go. Nature 2011;22:480487

13 Van der Greef J, van Wietmarschen H, Schroën J, Wang M, Hankemeier T, Xu G: Systems biology-based diagnostic principles as pillars of the bridge between Chinese and Western medicine. Planta Med 2010;76: 2036-2047.
14 Witt CM, Manheimer E, Hammerschlag R, Lüdtke R, Lao L, Tunis SR, Berman BM: How well do randomized trials inform decision making: systematic review using comparative effectiveness research measures on acupuncture for back pain. PLoS One 2012;7:e32399.

15 Witt CM, Aickin M, Cherkin D, Che CT, Elder C, Flower A, Hammerschlag R, Liu JP, Lao L, Phurrough S, Ritenbaugh C, Rubin LH, Schnyer R, Wayne PM, Withers SR, Zhao-Xiang B, Young J, Berman BM; Collaborators: Effectiveness guidance document (EGD) for Chinese medicine trials: a consensus document. Trials 2014;15:169.

16 Witt CM, Aickin M, Baca T, Cherkin D, Haan MN, Hammerschlag R, Hao JJ, Kaplan GA, Lao L, McKay T, Pierce B, Riley D, Ritenbaugh C, Thorpe K, Tunis S, Weissberg J, Berman BM; Effectiveness Guidance Documents Collaborators: Effectiveness Guidance Document (EGD) for acupuncture research - a consensus document for conducting trials. BMC Complement Altern Med 2012;12:148.

17 Woolf SH, Purnell JQ, Simon SM, Zimmerman EB, Camberos GJ, Haley A, Fields RP: Translating evidence into population health improvement: strategies and barriers. Annu Rev Public Health 2015;Epub ahead of print. 